\title{
Transmurality: Catch Me If You Can
}

Gopi Dandamudi MD FHRS, Krannert Institute of Cardiology at the Indiana University School of Medicine, Indianapolis, IN 46032

COI: Consultant, Medtronic Inc.

Word Count: 1167

\section{Correspondence:}

Gopi Dandamudi, MD FHRS

System Medical Director, Indiana University Health Electrophysiology

Medical Director, IUH Atrial Fibrillation Center

Indiana University School of Medicine

1801 N. Senate Boulevard

Indianapolis, IN 46202

Tel: 317-962-0105

Fax: 317-962-0501

Email: gdandamu@iu.edu

This is the author's manuscript of the article published in final edited form as:

Dandamudi, G. (2017). Transmurality: Catch Me If You Can. Heart Rhythm.

http://dx.doi.org/10.1016/j.hrthm.2017.02.034 
Transmurality of ablation lesions has always eluded us in the electrophysiology world. We walk a fine line between achieving permanent transmural lesions to increase efficacy at the expense of creating collateral damage. This is even more true in thin cardiac structures such as the left atrium and the pulmonary vein antra that are surrounded by collateral structures, including the esophagus, phrenic nerve, esophageal plexus and even coronary arteries such as the left circumflex artery. Significant and sometimes life threatening complications related to atrial fibrillation (AF) ablations have been reported over the years, including creation of atrioesophageal fistulas, gastroparesis, right phrenic nerve palsy, bronchial injury and even myocardial infarctions (1-4).

In the quest to find the right tools that delivers safety and efficacy, various energy sources and delivery mechanisms have been studied. Lesion science has been well studied by several authors over the years to understand mechanistic implications of our therapies. These have included various forms of energy, including radiofrequency, cryothermy, laser and ultrasound energy. Also, to accompany the energy sources, catheter designs have morphed to try to deliver contiguous and transmural lesions. Circular pulmonary vein ablation catheters, balloon mounted catheters using cryothermy, laser, and ultrasound energy have all been studied in the past with varying successes.

In this paper, Avital and colleagues report their findings using mini electrode (ME) mounted irrigated and standard ablation catheters (5). Specifically, in a canine model of chronic atrial fibrillation ( $>1$ year), they report their experience of using MEs mounted to a $4 \mathrm{~mm}$ irrigated and an $8 \mathrm{~mm}$ standard ablation catheter (BSc). They studied local electrogram (EGM) characteristics as recorded by the MEs and by standard bipolar configurations (tip-ring). 
They report findings on real time EGM amplitude reductions and associated frequency spectral shifts (not in real time) and how such information can be used to guide ablation in the atria to achieve transmurality without causing complications.

The $4.5 \mathrm{~mm}$ irrigated catheter had 3 MEs placed $1.5 \mathrm{~mm}$ from the tip with $1.2 \mathrm{~mm}$ spacing between each electrode. Tip to distal ring distance was $8.6 \mathrm{~mm}$. The $8-\mathrm{mm}$ standard ablation catheter had 3 MEs placed $2 \mathrm{~mm}$ away from the tip and a tip to distal ring distance of $11.5 \mathrm{~mm}$. Real time sampling of EGMs was performed using MEs and the standard tip to distal ring configurations using 10 second samples and the average of six lowest and highest EGMs. Frequency spectral analysis was performed offline with demonstration of leftward shift to lower frequencies in ablated tissue. When compared to standard tip to distal ring configuration, the ME EGMs showed the highest amount of EGM amplitude reduction and frequency spectral shifts (almost twice in MEs compared to the standard tip to distal ring configuration). The end-point of each lesion was maximal EGM reduction that was defined as no further EGM amplitude decrease for 5 seconds during RF application. Tissue pathology revealed $82 \pm 24 \%$ and $87 \pm 14 \%$ transmurality of the lesions with the $4 \mathrm{~mm}$ and $8 \mathrm{~mm}$ catheters, respectively. There was no collateral damage to surrounding structures seen in the 4 animals.

This study offers interesting insights into atrial lesion formation based on EGM reduction and frequency spectral shifts, and the importance of monitoring local EGMs to guide lesion delivery to achieve safe and effective results. Even in a chronic AF model with lower amplitude EGMs $(<1 \mathrm{mV})$, the authors demonstrated reliably reduction in EGM amplitudes and frequency spectral shifts. In this chronic model, they also demonstrated a high degree of transmurality (close to $90 \%$ ) and no associated complications. The presence of MEs at the 
catheter tip allow for recordings of higher density of surrounding tissue of interest and avoids recording summation EGMs of tissue distant to the ablation site as seen with the tip to distal ring configuration. More reliable calculations regarding near field EGMs and real time assessment of changes in the quality and quantity of these EGMs is a welcome tool to advance the science of AF ablation. Such an ablation catheter does increase our confidence in reliably assessing lesion quality and prevents us from using excess energy that can potentially lead to increased complication rates.

However, there are some limitations with this small study. This was performed in only 4 canines and larger studies will need to be done to show consistency of these findings. At least in some cases, the MEs may straddle healthy and scar boundaries and percentage reduction of EGMs and frequency spectral shifts may be influenced by reduction of EGMs of healthier tissue rather than scar tissue. The applicability to ventricular tissue needs to be tested as well. Contact with tissue is also an important characteristic and it is unclear if adding contact force to this type of catheter may yield similar results. Frequency spectral analysis was performed off line and enabling systems to allow online real time analysis is important for such technology to be adopted down the road. Even though the authors report no collateral injury with their ablation strategy in these canine models, the sample size is too small to draw meaningful conclusions and its applicability in humans stills needs to be tested.

In summary, the authors have highlighted in this study the utility of MEs in guiding ablation in a canine model of chronic AF and studying real time lesion characteristics in the form of near field EGM amplitude reductions and offline frequency spectral shifts. More effective and safer lesions can be delivered resulting in high degree of lesion transmurality. 
Such innovations are always welcome in the ever- growing field of AF ablation and will only add to our understanding of this highly complex disease. 


\section{References:}

1. Barbhaiya CR, Kumar S, John RM, Tedrow UB, Koplan BA, Epstein LM, Stevenson WG, Michaud GF. Global survey of esophageal and gastric injury in atrial fibrillation ablation. J Am Coll Cardiol 2015;65(13):1377-8.

2. Chugh A, Makkar A, Ho SY, Yokokawa M, Sundaram B, Pelosi F, Jongnarangsin K, Oral H, Morady F. Manifestations of coronary arterial injury during catheter ablation of atrial fibrillation and related arrhythmias. Heart Rhythm 2013;10(11):1638-45.

3. Sacher F, Monahan KH, Thomas SP, Davidson N, Adragao P, Sanders P, Hocini M, Takahashi Y, Rotter M, Rostock T, Hsu LF. Phrenic nerve injury after atrial fibrillation catheter ablation. J Am Coll Cardiol 2006;47(12):2498-503.

4. Verma N, Gillespie CT, Argento AC, Tomson T, Dandamudi S, Piña P, Ringwala S, Lin AC, Chicos $A B$, Kim S, Arora R. Bronchial effects of cryoballoon ablation for atrial fibrillation. Heart Rhythm 2017;14(1):12-6.

5. Avitall B, Kalinski A, Horbal P, Koblish J. The Relationship between Lesion Formation and Electrophysiological Responses Using Catheters Equipped with Mini Electrodes in Chronic Atrial Fibrillation. Heart Rhythm 2017 